\title{
Synchronous Big Data Analytics for Personalized and Remote Physical Therapy
}

\author{
Prasad Calyam, Anup Mishra, Ronny Bazan Antequera, Dmitrii Chemodanov, \\ Alex Berryman, Kunpeng Zhu, Carmen Abbott, Marjorie Skubic \\ Center for Eldercare and Rehabilitation Technology, University of Missouri-Columbia, USA; The Samraksh Company \\ Email: \{calyamp, skubicm\}@missouri.edu, \{akmm94,rcb553,dycbt4\}@mail.missouri.edu, \{alex.berryman, tony.zhu\}@ samraksh.com, \\ abbottc@health.missouri.edu
}

\begin{abstract}
With gigabit networking becoming economically feasible and widely installed at homes, there are new opportunities to revisit in-home, personalized telehealth services. In this paper, we describe a novel telehealth eldercare service that we developed viz., "PhysicalTherapy-as-a-Service" (PTaaS) that connects a remote physical therapist at a clinic to a senior at home. The service leverages a high-speed, low-latency network connection through an interactive interface built on top of Microsoft Kinect motion sensing capabilities. The interface that is built using user-centered design principles for wellness coaching exercises is essentially a 'Synchronous Big Data' application due to its: (i) high data-in-motion velocity (i.e., peak data rate is $\approx \mathbf{4 0 0} \mathrm{Mbps}$ ), (ii) considerable variety (i.e., measurements include 3D sensing, network health, user opinion surveys and video clips of RGB, skeletal and depth data), and (iii) large volume (i.e., several GB of measurement data for a simple exercise activity). The successful PTaaS delivery through this interface is dependent on the veracity analytics needed for correlation of the real-time Big Data streams within a session, in order to assess exercise balance of the senior without any bias due to network quality effects. Our experiments with PTaaS in an actual testbed involving senior homes in Kansas City with Google Fiber connections and our university clinic demonstrate the network configuration and time synchronization related challenges in order to perform online analytics. Our findings provide insights on how to: (a) enable suitable resource calibration and perform network troubleshooting for high user experience for both the therapist and the senior, and (b) realize a Big Data architecture for PTaaS and other similar personalized healthcare services to be remotely delivered at a large-scale in a reliable, secure and cost-effective manner.
\end{abstract}

Index Terms-Smart Health Care, Personalized Remote Physical Therapy, Synchronous Big Data, Gigabit Networking App

\section{INTRODUCTION}

Particularly in the case of eldercare, health and wellness services can be proactively delivered from remote clinic sites to allow unobtrusive, continuous monitoring and personalized coaching in homes of seniors. Given the challenges being faced in providing smart health care services due to limited facilities as well as human resources to large geographically distributed senior populations, a new class of telehealth services are essential to deliver healthcare on a realtime basis. With Gigabit networking becoming economically feasible and widely installed in homes through city-supported programs such as Google Fiber in several US cities (e.g.,

This work was supported by National Science Foundation under award numbers: CNS-1346789, ACI-1246001 and ACI-1245795, and the Department of Energy under award numbers: DE-SC0001331 and DE-SC0007531. Any opinions, findings, and conclusions or recommendations expressed in this publication are those of the author(s) and do not necessarily reflect the views of the united states government or any agency thereof.
Kansas City), there are new opportunities to revisit in-home, personalized telehealth services. The opportunities include the conceptualization and implementation of Gigabit applications that use the latest advances in sensing, high-definition videobased communication, and cloud computing. The Gigabit applications within novel telehealth service compositions can enable: early interventions, better health outcomes through fewer hospital visits, better one-on-one interactive care, and ultimately significant cost savings in the relatively expensive eldercare health market sector.

In the diverse field of eldercare, novel telehealth services in the context of "Physical Therapy" are particularly attractive for both hospitals and senior patients. Physical and cognitive limitations of seniors as they age can present difficulties for seniors to travel to a physical therapy clinic for on-going interactions with a therapist. In this paper, we describe a novel telehealth service that we developed viz., "PhysicalTherapyas-a-Service" (PTaaS) that connects a remote physical therapist in a clinic to a senior at their private home.

The system design and components of the service leverage a high-speed, low-latency network connection through an interactive interface built on top of Microsoft Kinect motion sensing capabilities. We utilize the openly available Kinect Application Programming Interface (API), C\# language and Windows Presentation Foundation (WPF) technologies on .Net Framework 4.5 in our interactive interface development environment. The service hardware components in addition to the Kinect sensors include measurement point appliances for application and network monitoring services provided by Narada Metrics [1]. We also use a cloud-hosted storage environment provided by Global Environment for Network Innovations [2] for synchronous analytics of the data streams in the PTaaS session. Two separate interface variants have been developed, one for the therapist side, and another for the senior side, in order to address the unique needs of the visual interaction (e.g., the therapist can use voice commands) and exercise assessment (e.g., the therapist can generate exercise activity reports for discussion with the senior).

Our PTaaS interface that is built with user-centered design principles for in-home senior directed health and wellness coaching exercises is more than a typical videoconferencing application. It integrates $2 \mathrm{D}$ video feeds between the therapist and senior along with 3D sensing data to provide an immersive experience, and thus is essentially a 'Synchronous Big Data' application. The Big Data characteristics are due to its: (i) high data-in-motion velocity (i.e., peak data rate is $\approx 400$ Mbps and average is over $100 \mathrm{Mbps}$ ), (ii) considerable variety (i.e., measurements include 3D sensing, network health, user 
opinion surveys and video clips of RGB, skeletal and depth data), and (iii) large volume (i.e., several GB of measurement data for a simple exercise activity).

The successful PTaaS delivery through this interface is dependent on the veracity analytics needed for correlation of the real-time Big Data streams within a session, in order to quantitatively assess balance of the senior without any bias due to network quality effects. More specifically, a therapist needs to be able to confidently assess whether non-ideal performance in the exercise forms of a senior is being impacted due to lag in network communications for the data-intensive interactive session, or in fact are due to the physical and cognitive limitations of the senior owing to aging. Our analytics approach involves a network quality estimation module whose output (i.e., green or red indicator on the interface for network quality status) provides visual evidence to the senior and therapist regarding any undesirable network status that degrades interface user experience and may cause any exercise mis-assessment.

We investigate our synchronous Big Data nature of our PTaaS interface through experimental testbeds, both in a laboratory environment as well as in an actual testbed involving senior homes in Kansas City with Google Fiber connections and our university clinic site in Columbia, Missouri. Our experiment results clearly demonstrate the network configuration and time synchronization related challenges in order to perform online analytics on the data streams in a PTaaS session for different physical therapy activities such as balance, sway detection, and analysis of walking gait patterns. Our PTaaS interface test plan and experiment results discussion are focused around the synchronous big data analytics for ideal network quality condition scenarios, and bad network health quality scenarios. We also qualitatively study how an immersive telehealth setup for physical therapy with our PTaaS interface compares with a face-to-face session, and explore the pros and cons of our approach from user experience and 'therapy effectiveness' standpoints.

Our findings from the PTaaS interface development and testbed experiments in this paper provide insights on how to: (a) enable suitable resource calibration and perform network troubleshooting to achieve good user experience for both the therapist and the senior, and (b) realize a Big Data architecture for PTaaS and other similar smart healthcare services to be delivered at a large-scale in a reliable, secure and cost-effective manner. Thus, our work explores a relatively less explored research problem of combining analytics of telehealth user experience in conjunction with Big Data model characterization of a Gigabit application that is heavily network resource intensive, while also being highly sensitive to network lags. It also represents an innovative and novel approach of using broadband and cloud technologies for 'proactive' healthcare that is direly needed to minimize the rising health care costs in our society.

Our PTaaS development efforts are part of our engagement within the vibrant Gigabit application developer community that is growing through initiatives such as Mozilla Ignite and US Ignite [3] being supported by the National Science Foundation within the USA. Specifically, the US Ignite organization is fostering teams to develop the next-generation of Internet applications that provide transformative public benefit in the areas of national priorities such as healthcare, public safety and education.
The remainder of this paper is organized as follows: Section II describes related work. In Section III, we formally describe the PTaaS interface components and the synchronous Big Data as well as high-speed network connectivity issues involved in our telehealth service development. Section IV details our human subject testing methodology and qualitative results within actual testbeds. Following this in the same section, we also present controlled network results from our synchronous Big Data analytics. Section V outlines a Big Data architecture for PTaaS for large-scale delivery. Section VI concludes the paper.

\section{RELATED WORK}

There have been earlier studies on remote physical therapy and other exercise systems. In [4], two different virtual reality applications are proposed using Vicon and Kinect technologies, for both local and remote physical therapy. Avatars of the patient and the therapist are created in real-time within the applications, and the communications between the patient and therapist is through each other's Avatar models. In another related study [5], a motion capture application is proposed for remote orthopedics rehabilitation. This system uses a set of gaming motion capture technologies (e.g., Wii, Kinect, and Playstation Move) for exercise data collection in different scenarios involving clinic and home sites. In remote sessions, the exercise data are not exchanged in real-time through a videoconferencing setup, but rather are sent over a network for offline (not real-time) visualization and feedback. The offline nature was pertinent in their case given the purpose of their application was to allow a patient to learn how to perform exercises correctly at the hospital, and later execute them at their home without any therapist by their side. Other works have investigated exercise coaching with vision through a socially assistive robot coach [6], pre-recorded videos with automated coaching advice [7], the Wiimotes attached to limbs for movement-training exercises [8], and wearable sensing with iPods for tracking home balance exercises [9].

In contrast to these works, our PTaaS application approach involves a peer-to-peer communication model for sending and receiving skeletal data (i.e., $2 \mathrm{D}$ skeletal visual showing joint locations) as well as RGB, depth, and audio data in real-time, all using a high-speed network setup in a wide-area setting. Hence, our approach aims to establish a more realistic and highly interactive/immersive communication between therapist and patient in the comfort of a clinic and private home, respectively.

In terms of remote access to video streams, earlier works have proposed techniques to perform snapshot captures for streaming over low-bandwidth network paths [10]. In addition, real-time video compression schemes for users connected to access networks with high latency and different available bandwidth levels have been proposed [11]. In [12], authors present network traffic prioritization and load balancing schemes to enable Skype sessions over portable devices with satisfactory user experience for telehealth applications such as videoconsultation-call in a home area network. Our application design goals are different and we aim for video streams to be delivered without compression over wide-area network paths with low-latency characteristics, and take advantage of highspeed Gigabit networking between patient homes and a remote therapist clinic. Our network-awareness work builds upon the 


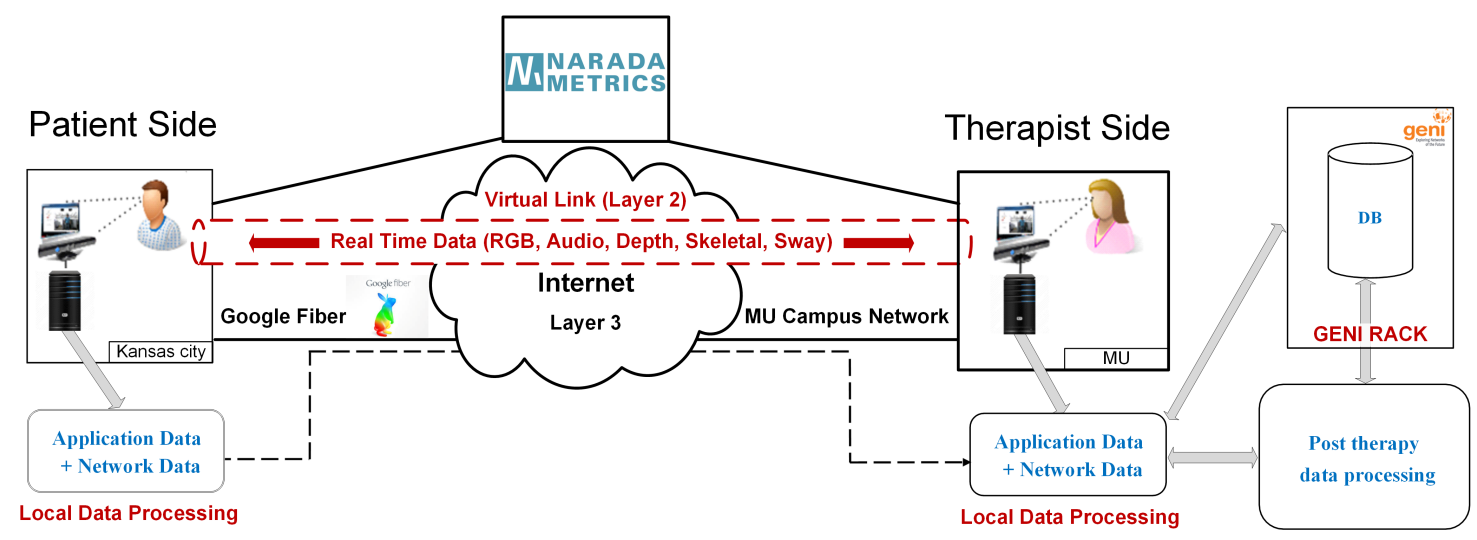

Fig. 1: Physical Therapy-as-a-Service System Setup

earlier work in [13], where real-time network measurements are used for notifying users regarding network quality status in order to guide them in their real-time interactions with remote scientific instruments. The difference in this work is that we use the network quality status to help improve the remote physical therapy effectiveness in a interactive videoconferencing context supplemented by $3 \mathrm{D}$ sensor data.

Our PTaaS application requires on-line and post data analysis of synchronous Big Data within a remote physical therapy session. Our literature survey indicated that there are few works such as [14] and [15] that deal with synchronous Big Data analytics with applications data streams. The authors in [14] analyze social network data and show how interactive Big Data analysis can help formulate and assess hypotheses in a rapid and iterative manner. The work in [15] considers distributed system optimization strategies to enable synchronous parallel processing of Big Data streams within a cloud platform. Following the definition in [16], these works as well as our work can be viewed as Big Data due to the nature of the data that pushes us to look beyond triedand-true methods that are in the current state-of-the-art. We basically use the common data analysis steps of: (i) integrating, cleaning and transforming data, and (ii) actual data analysis with aggregation and/or visualization - in order to solve unique analytics challenges. Our unique analytics challenge can be seen in the time synchronization issues within the data streams in order to real-time align the various activities between the therapist and the patient. Our PTaaS data streams analysis methodology correlates the perceived user experience with the various network quality metrics for different video stream configurations (i.e., skeletal, RGB, depth) with synchronized audio for helping the therapist verify the balance assessment hypotheses without excessive processing delays.

It has been observed in earlier studies such as [17] and [18] that sensing data from Kinect is prone to accuracy errors. The errors relate to image distortions in the data capture processes due to the factory pre-calibration issues that specially impact at the depth data-type level. To counter this problem and increase accuracy in the data capture process, the authors in [17] rely on indoor control field to calibrate Kinect sensor along with the accurate internal parameters of source and destination side cameras and their relative pose. In [18], authors handle the errors using a method that involves modeling the intrinsic and extrinsic parameters of the distortions in the depth image data.
In our PTaaS application context, these accuracy errors do not significantly impact effectiveness of the remote physical therapy sessions because our interactive interface application does not heavily rely on depth data accuracy. For proper functioning of our interactive interface application, the RGB and Skeletal data quality and accuracy are more critical to minimize errors due to miscommunication in those data types. Also, our focus on RGB and Skeletal data addresses the need for the physical therapist to capture the real representation of the subjects, timelines of interaction and follow-through when performing exercises. Moreover, in our recent work [19], our experiments with the PTaaS application in a controlled setting demonstrated that Kinect skeletal models offer acceptable accuracy for use in a remote physical therapy system setting.

\section{PhysicAl TherAPY-AS-A-SERVICE (PTAAS)}

In this section, we first present the PTaaS application requirements and system overview. Following this, we detail the salient components of the PTaaS system and describe the user-centered design principles. Next, we show how the PTaaS interactive interface is essentially a synchronous Big Data application. Lastly, we describe the importance of high-speed network connectivity for our PTaaS system and methods to reduce peak data rates for lower resolution modes of operation.

\section{A. PTaaS System Overview}

Figure 1 shows the system design for the immersive interactions between the Therapist and the Patient using a network overlay setup based on virtual link (Layer 2) technologies. The overlay path is needed for fast data movement of video, audio, RGB, depth and skeletal data for real-time display of gait and other movement parameters at both ends. Given that private home $1 \mathrm{Gbps}$ access network connections of Google Fiber customers in Kansas City (or any other Internet Service Provider) do not have public IP addresses, peer-topeer networked applications such as our PTaaS interactive interface needs to rely on a overlay setup to bind the ports between the Therapist and Patient applications, and to allow custom protocol communications. On each side, the interactive interface uses a Kinect device along with a local computer (specifications: Windows 764 bits, RAM 4 GB, HDD 500GB, Gigabit NIC) that is mounted on a mobile cart that has a large display (specification: 1920x1080 px). 


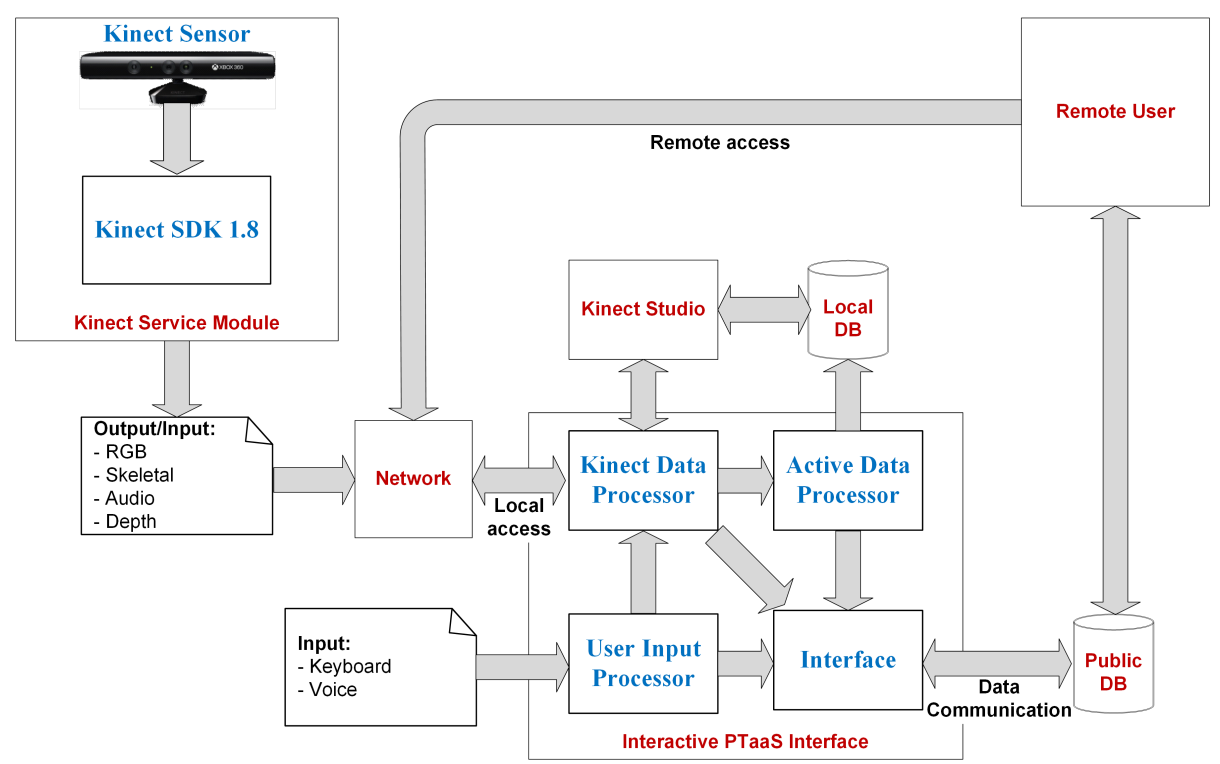

Fig. 2: PTaaS Interface Architecture and Components

For the out-of-band communications to support data processing with session-related data logs, we utilize regular Internet (Layer 3) protocols. The application and network data on both sides are processed locally for tasks such as preliminary sanitization and meta data annotation (e.g., data folder naming), and are immediately sent to a cloud-hosted database for detailed data processing that help with later activity report discussions between the Therapist and the Patient. We utilized the GENI Rack at University of Missouri (MU) for the database, and also hosted the peer-to-peer application orchestration signaling co-ordination module also within the GENI Rack that is part of the overlay setup. For these purposes, the GENI Rack was configured with three virtual machines provisioned using the ESXi hypervisor. Last but not the least, the entire application and network measurement data collection and aggregation as well as visualization are performed with the Narada Metrics software [1] that we have previously developed as a end-to-end measurement framework.

\section{B. PTaaS Components}

1) Interactive Interface: Figure 2 shows the PTaaS interface related software architecture, and the various components that use the Microsoft Kinect motion sensing capabilities. We utilize the openly available Kinect Application Programming Interface (API) within the Kinect SDK v1.8, C\# language and Windows Presentation Foundation (WPF) technologies on .Net Framework 4.5 in our interactive interface development environment. The main component that connects the Kinect sensor to our application is the 'Kinect Service Module' that collects the various data sets during the session, and writes it to the network interface for both local and remote access. The individual data streams on both the sender-side and receiver-side are accessed using the corresponding network IP addresses and respective port numbers. The remote access enables the interactive videoconferencing feature of the interface, whereas the local access enables the preliminary processing and the data export into the public cloud-hosted database. For the local processing of raw Kinect data within the 'Kinect
Data Processor' component to analyze the saved activities and generate information for data correlations, we utilize the file formats and processing features such as 'rewind' and 'play' of the Kinect Studio software.

The Therapist and Patient each have a slightly different software configuration in order to suit the respective userspecific requirements. The screenshots of the 'Interactive PTaaS Interface' that we developed for the Therapist and Patient sides are shown in Figure 3. The Therapist side has additional real-time display of detailed sensing and network quality data to help with the exercise assessments. To enable convenient administration of exercises, the Therapist side interface has support for voice commands and keyboard-mouse input handling that allows for start/stop markers within data between exercise activities. Note that the Therapist sees a larger scale frame of the Patient's view, shown on the left panel; whereas, the Patient sees both sides views in a equal sized panels side-by-side. The bottom right shows the skeletal data visualization within the session. The network strength status bar is either 'green' or 'red' in color based on the output of the network quality estimation module within the 'Active Data Processor' explained in the following sub-section. This visual format was designed through multiple experiments involving users, and by gathering feedback and refining the interface over multiple iterations during the development stage. Overall, this visual format of the interactive interface helps with effective involvement of both the Therapist and the Patient within the session, and provides a convenient method to administer the different exercise activities.

2) Network Quality Estimator: Given that a robust network architecture with predictable end-to-end performance is essential for the interactive monitoring and coaching in the PTaaS session, we deeply instrumented the PTaaS system to obtain measurements for network quality estimation. Both active measurements (i.e., end-to-end TCP throughput, roundtrip time (RTT) delay, jitter and packet loss) and passive measurements (i.e., transfer rate on uplink of the local interface, download rate at remote interface) were collected using the Narada Metrics measurement framework. 


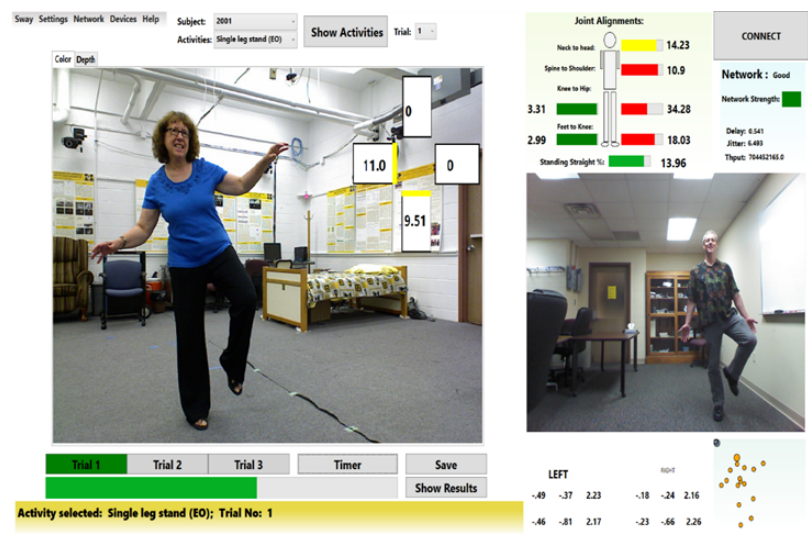

(a) Therapist View

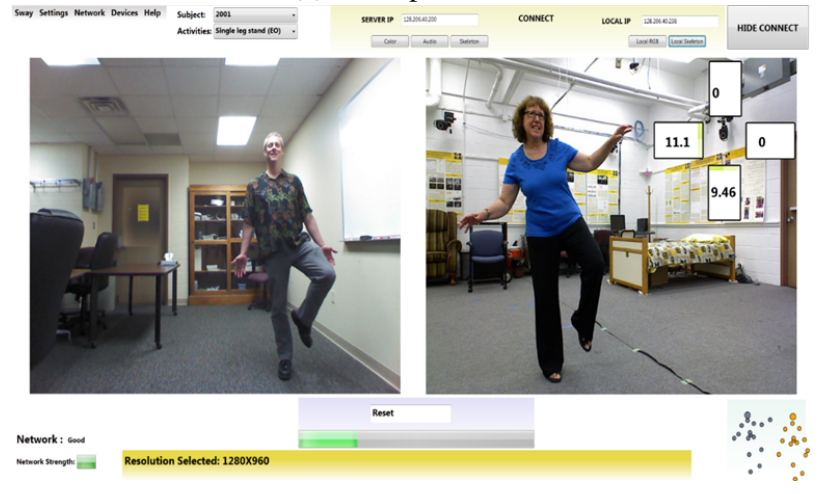

(b) Patient View

Fig. 3: PTaaS Interactive Interface Screenshots

We scheduled conflict-free active measurement tests with Iperf and Ping tools using the Narada Metrics active measurement scheduler [1]. Iperf tool tests that are computation and channel intensive are performed offline in a periodic sampling manner with inter-sample times of 30 minutes to 1 hour, before the use of the PTaaS application on a network path. However, the lightweight Ping tool tests are performed inline with the PTaaS application with a sampling rate of 30 seconds. The passive measurements were in-line with the application traffic and were collected using the popular Wireshark packet capture tool, as well as the 'Windows Network Interface' counters viz., BytesReceivedPersec and BytesSentPersec. In order to keep the measurement tap to be non-intrusive, we experimented with different settings of the sampling period of the interface statistics, and chose a sampling rate of 10 seconds within the PTaaS interface.

The raw measurement data size for a simple session was several GB for the active and passive measurements. We stored all of the summarized measurement data in JSON format and allowed for publish (from the Interface to send raw measurements) and subscribe (from the Interface to request processed/correlated measurements) within a session. In addition, detailed metrics information, graphs and other drilldown features for measurement data analysis were provided through a customized Narada Metrics Dashboard shown in Figure 4. The measurement analysis through Narada Metrics greatly helped in the resource provisioning and end-to-end performance monitoring for the PTaaS system and helped overcome several performance bottleneck challenges. For instance, in a case relevant to network resource provisioning, we

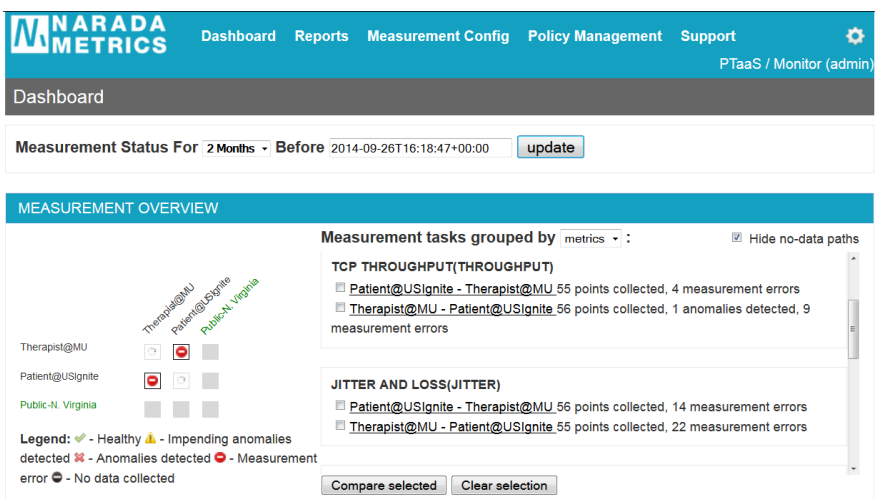

Fig. 4: Customized Narada Metrics Dashboard for PTaaS

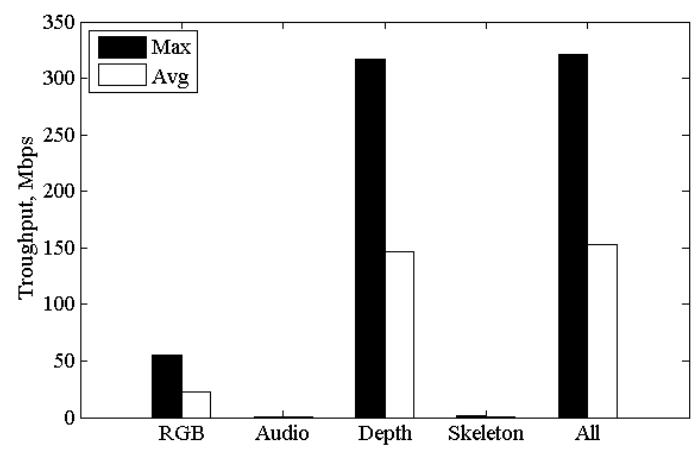

Fig. 5: Throughput of different PTaaS Interface Modes

were able to characterize how much bandwidth is consumed by the PTaaS interface for four application data streams: RGB, Skeleton, Audio and Depth. We were able to observe that the Skeleton and Audio data have minimal bandwidth consumption, the RGB data has medium bandwidth consumption, and the Depth data was a heavy consumer of network bandwidth consumption as shown in Figure 5.

In a different case relevant to performance troubleshooting, using a "Patrol App" customization across multiple vantage points on the wide-area network with a unified dashboard of the various metrics across locations, we were able to identify optimal and often suboptimal application configurations in sessions with various homes. As shown in Tables I and II, we were able to detect actual deployment scenarios where bottlenecks due to mis-configuration of network ports (due to duplex mis-match of Ethernet interfaces) that degraded the interactivity and videoconferencing quality.

Based on the various measurement functions supported within Narada Metrics, we are developing an intelligent 'Network Quality Estimator' module within the PTaaS system that is able to aggregate and correlate the various active and passive measurements of application and network performance. This will allow us to provide tangible performance intelligence to the PTaaS interface, and ultimately to the Therapist for making proper exercise form assessments. Our network quality estimator at the basic form can be represented as a neural network as shown in Figure 6. Considering only the active measurements, there are four dimensions of end-to-end network path performance that include: throughput or available bandwidth $B_{a v}$, delay $D$, losses in the network path $L$ and 
TABLE I: MU Therapist side performance gathered from Narada Metrics

\begin{tabular}{|c|c|c|c|c|c|c|}
\hline Activity under Test & Available Bandwidth, Mbps & Jitter, mlsec & Loss, \% & RTT, mlsec & Upload Rate, Mbps & Download Rate, Mbps \\
\hline Single leg stance & 3.91 & 14.53 & $\mathbf{7 3 . 8 4}$ & 30.67 & $\mathbf{0 . 3 4}$ & $\mathbf{1 . 9 4}$ \\
\hline
\end{tabular}

TABLE II: Kansas City Senior side performance gathered from Narada Metrics

\begin{tabular}{|c|c|c|c|c|c|c|}
\hline Activity under Test & Available Bandwidth, Mbps & Jitter, mlsec & Loss, \% & RTT, mlsec & Upload Rate, Mbps & Download Rate, Mbps \\
\hline Single leg stance & 4.9 & 6.30 & 0.86 & 15.75 & 1.3 & 3.16 \\
\hline
\end{tabular}

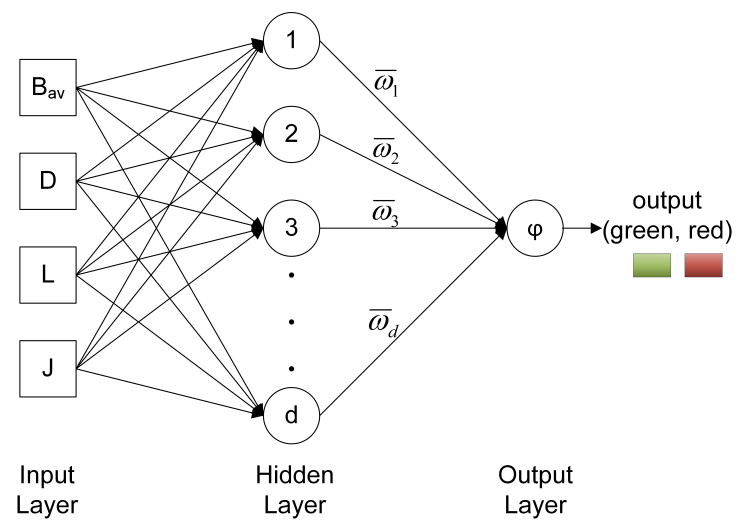

Fig. 6: Model of Network Quality Detector

packet jitter $J$. Our neural network model for the active measurements can be expressed mathematically as:

$$
\phi=\phi\left(w_{i, B} \cdot B_{a v}, w_{i, D} \cdot D, w_{i, L} \cdot L, w_{i, J} \cdot J\right)
$$

where $w_{i, B}, w_{i, D}, w_{i, L}, w_{i, J}$ are coefficients of different application modes $(i=1, d)$. If $\phi>0$, we can say that network is not a factor in the assessment of Patient exercise performance. The opposite is true (i.e., network is a degrading and misleading factor for Patient exercise performance assessment) for $\phi<0$; Note that $\phi=0$ is the baseline and $\phi$ can be any of the different functions such as sign or tanh. Our approach is to construct such individual models for different measurement data sets at the application and network levels, and use a combined closed-form mathematical expression that can predict the network quality status for any given inputs of measurements in an actual deployment. Note that exact solution of such an approach is outside the scope of this paper and is part of our future work. With regards to our current work, our network quality estimator uses a set of thresholds based on empirical performance history to output either a 'green' or 'red' status signal displayed on the PTaaS interface in the 'Network Strength' panel shown in Figure 3.

\section{Synchronous Big Data Model}

The network bandwidth consumption results in Figure 5 show how the PTaaS interface has high velocity when there is application data-in-motion. We have consistently observed peak data rates of $\approx 400 \mathrm{Mbps}$ and average data rates of over 100 Mbps for simple exercise activity tasks with the PTaaS interface between an actual Therapist and Patient in a closednetwork setting. In addition, given the considerable variety of data involved in a PTaaS session such as 3D sensing, network health, user opinion surveys and video clips of RGB, skeletal and depth data - we can appreciate the various quantitative and qualitative information that needs to be correlated to make sense of the data. The user opinion surveys form more of the qualitative data of user's quality of experience (QoE), however we can adopt a 'Mean Opinion Score' (MOS) ranking scale of $1-5$, where 1 rating is Poor and 5 is Excellent.

Further, the data volumes we have seen in a PTaaS session are quite high, and are several GB of measurement data for a simple exercise activity. For instance, 1 second of video at a resolution of $1280 \times 960$ pixels for depth at 30 frames per second generates approximately $140 \mathrm{MB}$ of data. Given that a typical PTaaS session lasts for several tens of seconds or even minutes as shown in Figure 7, the data generated quickly can exceed several GB of measurement data. If we configure the PTaaS interface to collect gait, bed and motion sensor data to extend it for home fall-detection and gait analysis and health change alerts as done in previous work [20]- [22], the data volume can be significantly large and again on the order of several tens of GB (we noticed worst case data collection can be $\approx 150$ GB per hour).

Based on the above discussion, we can argue that the realtime PTaaS interface requirements, and application resource consumption behavior can be characterized as being of the 'Synchronous Big Data' nature. Consequently, it requires us to integrate new methods to integrate, clean and transform data that is in different scales and is collected at different sampling rates (or frequencies) for actual data analysis with aggregation and/or visualization. Moreover, our need to solve unique analytics challenges described in Section II is a guideline for the veracity needed within our data analysis for correlation of the real-time Big Data streams within a session for different time frames, in order to quantitatively assess balance of the senior without any bias due to network quality effects. As shown in Figure 7, the time frames can relate to different exercise forms such as Single leg stance (eyes open), Tandem walk (eyes open), and Tandem walk (eyes closed). We need to divide our analytics for different time frames depending on the data nature, and use significant computation power to look at meaningful ways to query data and correlate the individual analyses to find any network effects that impact the PTaaS exercise assessments, e.g., when a Therapist observes a Patient is having balance problems during the exercises.

\section{Importance of High-speed Network Connectivity}

Herein, we discuss the importance of having a high-speed bandwidth, low latency network connection for our PTaaS application. We have found that a high-speed network connection is a critical need for proper usability of our interactive interface, and to minimize the patient risk due to miscommunication of exercise activity with the therapist. In addition, the large quantity of data generated by our PTaaS application 
in the order of severe tens of GB just within a single PT session requires us to use a robust and high-speed networking infrastructure for real-time data import into a remote database for synchronous Big Data analytics explained in the previous subsections. However, we have explored a few methods to reduce the peak data rate and average data needed by our interactive interface application modes of operation. In our current application implementation, our PTaaS application can be used in a low resolution mode, and switched a high resolution mode if enough end-to-end session network bandwidth is available. We can also reduce the peak data rate if we turn off the depth data transmission in the interactive interface application, when there are network bandwidth limitations. We consciously do not compress the video or reduce the Frames Per Second because we need to deliver high-quality video to show detailed movements and facial expressions in the session between the Therapist and Patient. Another reason we do not compress the video is our original intent in this study to fully utilize the new paradigm of Gigabit network connections to residential homes through offerings such as Google Fiber, and thereby explore benefits for immersive PTaaS application delivery.

\section{Personalized Patient-Centric Study}

In this section, we first describe our human subject selection and the testing methodology within actual testbeds. Following this, we present controlled network results from our synchronous Big Data analytics that shows the network configuration and time synchronization related challenges for different physical therapy activities and interface resolutions.

\section{A. Testing Methodology}

We recruited 5 healthy senior participants from 4 different senior homes in the Kansas City areas where Google Fiber access network connections were available. Participants (our human subjects) were chosen to represent those who would benefit from a smart remote in-home physical therapy system as part of wellness coaching activities. Fortunately, the Missouri's regional backbone network operated by MOREnet was directly peering with Google Fiber, and our MU campus was connected at 1 - $10 \mathrm{Gbps}$ speeds on different network interfaces to MOREnet. Thus, we were uniquely positioned to conduct different experiments with our Gigabit application on actual testbed settings and with real users.

We were assisted by a professional Physical Therapist on the MU campus in Columbia, Missouri to define the exercise protocols that involve upper body and lower body motion for the PTaaS sessions, and to work with the seniors and our PTaaS interface in our testing activities. Precautions were taken in the protocol to limit any risks such as physical or cognitive injury in the human subject interactions with the PTaaS interface. We strictly followed our campus IRB approved guidelines in our recruitment and discussions related to test plan administration with the 5 human subjects.

The main testing goals were related to evaluation of the usability, user satisfaction and therapy effectiveness on both the Therapist and the Patient sides using a survey shown in Table III. More specifically, we are interested in studying how well the Therapist is able to effectively evaluate the various survey categories, and how well the seniors are able to follow the Therapist instructions when using the highdefinition videoconferencing feature of our PTaaS interface.
We considered requirements that are needed for care coordination in response to health issues such as the need for the Therapist to be able to see high-resolution skin color, facial expressions and fine-level motion trends of the remote senior.

\section{B. Qualitative Usability Results}

We conducted two sets of experiments, and asked the Therapist and the study participants representing the Patients to fill out the PTaaS user survey forms. In the first experiment set, we were interested in qualitatively studying how our 'PTaaS interface with 3D sensing data as well as videoconferencing capabilities' would compare to 'plain 2D video chat or even just with a telephone connection'. The Therapist was able to unequivocally conclude that just audio or audio-video $2 \mathrm{D}$ in real-time data is not very helpful for remote physical therapy related performance evaluation of a Patient. The 3D depth sensor data using Microsoft Kinect and the user-centered panel design in our PTaaS can provide important quantitative data such as 3D joint locations and joint angles to calculate gait and other performance parameters accurately. We explicitly remark that the accurate parameters information is critically needed to make a remote physical therapy session more informative about body dynamics of a Patient and can truly be effective for wellness coaching through better monitoring of Patient status. It also provides the Therapist with a richer ability to offer verbal, auditory and visual cues to perform correct movements within exercise forms.

In the second experiment set, we were interested in qualitatively studying the effectiveness of 'remote physical therapy' versus 'in-person physical therapy' when Patients perform in static and dynamic balance programs. Both the Therapist and the Patient concluded that both configurations were equally effective. However, given the technology advantages in obtaining more quantitative information regarding the Patient exercise performance in the PTaaS session, the Therapist concluded that the remote physical therapy session with a robust high-speed network connection could provide a truly immersive user experience. Experiment configurations with regular Virtual Private Network (VPN) software packages led to unusable sessions, and were rejected for use by both the Therapist and the Patients.

The Therapist also noted that audio quality was a very important feature, and any issues with feedback, background noises or echo could make the remote physical therapy session a failure, even if all of the visual elements had no impairments. The video quality during a remote physical therapy session with PTaaS interface was found to be affected due to the contrast of background or floor color, Patient or Therapist's clothing color, room lighting conditions, and network quality. One of the salient conclusions was that the users involved in a remote physical therapy session are advised to wear clothing (especially footwear) that is contrasting to the background or floor color for more optimal user experience and therapy effectiveness.

In the in-person physical therapy, accurate visual recognition of personal movements is harder without objective measurement devices, and thus remote physical therapy with devices such as the Kinect was more advantageous. Further, the Therapist preferred the remote physical therapy over inperson physical therapy in cases where the Therapist needed 


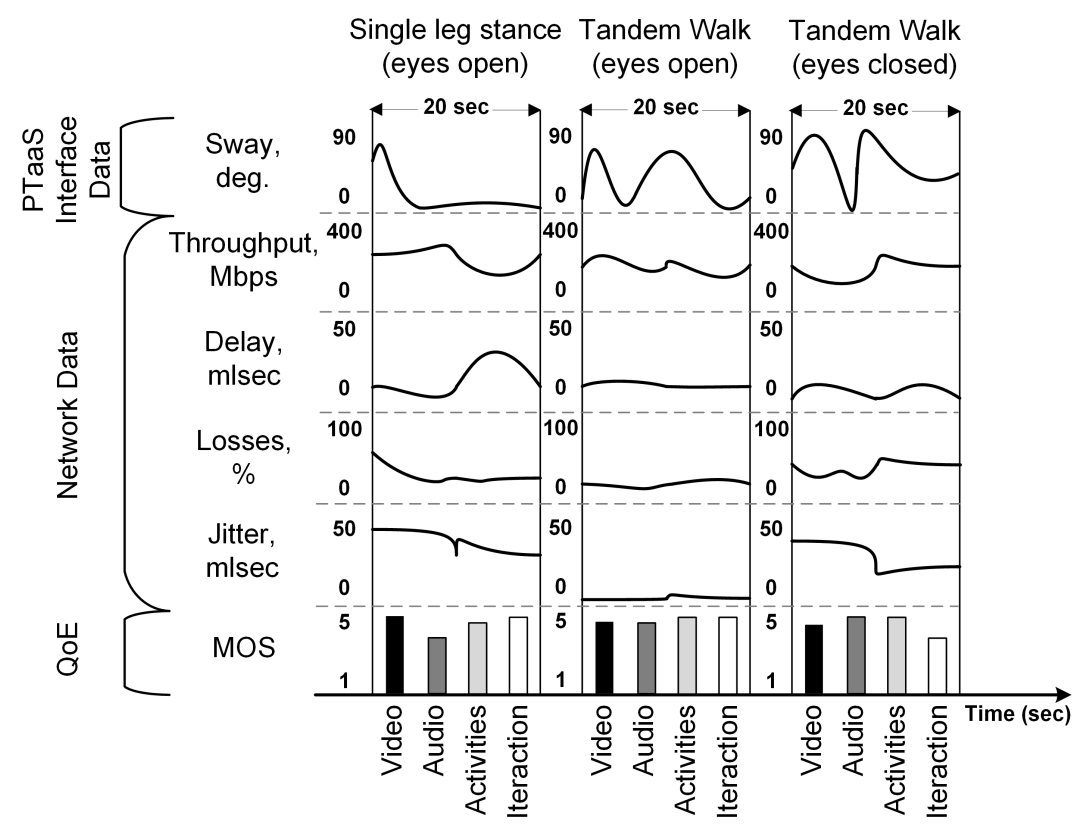

Fig. 7: Synchronous Big Data representation

TABLE III: PTaaS User Satisfaction and Interaction Effectiveness Survey Considerations

\begin{tabular}{|c|c|}
\hline \multicolumn{2}{|r|}{ A. Technical Quality of Video (rate 1/poor to 5/excellent) } \\
\hline 1. & $\begin{array}{l}\text { Video artifacts: Look for video artifacts around the remote users head and shoulders (e.g., blocks, image distortions such as tiling, or } \\
\text { out-of-focus areas). }\end{array}$ \\
\hline 2. & Sharpness: Observe whether object edge details and fine lines can be distinguished. \\
\hline 3. & Contrast, brightness, and color saturation: Observe whether the images are too dark or too white with a washed out appearance. \\
\hline 4. & Color depth: Look for color banding in the backgrounds and on the remote users faces. \\
\hline 5. & Stability: Evaluate whether images are stable with no motion in the background due to video artifacts, or video jitter noise. \\
\hline 6. & Background Clarity: Evaluate whether the background is out of focus; whether it is rich in color and texture. \\
\hline \multicolumn{2}{|r|}{ B. Technical Quality of Audio (rate 1/poor to 5/excellent) } \\
\hline 1. & Audio Clarity: Evaluate how clear the audio is, and whether echo noise occurs. \\
\hline 2. & $\begin{array}{l}\text { Audio Stability: Evaluate whether the audio quality is consistent or whether dropout interruptions or other audio degradation occurred, } \\
\text { including audio and lip movement being out of sync. }\end{array}$ \\
\hline \multicolumn{2}{|r|}{ C. User Satisfaction and Effectiveness (rate 1/strongly disagree to $5 /$ strongly agree) } \\
\hline 1. & I am able to satisfactorily use the video conferencing system with confidence. \\
\hline 2. & The video conferencing system is readily available and can connect to remote sites of interest. \\
\hline 3. & The video conferencing system is easy to use. \\
\hline 4. & I can communicate effectively to complete an agenda with the remote person using video conferencing. \\
\hline \multicolumn{2}{|r|}{ D1. Remote Interaction (rate 1/strongly disagree to 5/strongly agree) *Physical Therapist } \\
\hline 1. & My movements are accurately represented in the interactive coaching interface. \\
\hline 2. & I am able to see the movements that the remote senior user is performing. \\
\hline 3. & The remote senior user is able to follow my demonstrated movements. \\
\hline 4. & I am able to see how my movements compare to the movements of the remote senior user. \\
\hline 5. & I would be interested in using this system in the future for my clients who need rehabilitation services. \\
\hline \multicolumn{2}{|r|}{ D2. Remote Interaction (rate $1 /$ strongly disagree to $5 /$ strongly agree) *Senior } \\
\hline 1. & My movements are accurately represented in the interactive coaching interface. \\
\hline 2. & I am able to see what movements the remote physical therapist is demonstrating. \\
\hline 3. & I am able to perform the movements that the remote physical therapist is demonstrating, to the best of my ability. \\
\hline 4. & I am able to see how my movements compare to the movements of the remote physical therapist. \\
\hline 5. & I would be interested in using this system in the future if I have a need for rehabilitation services. \\
\hline
\end{tabular}

to compare self to remote Patient's movements. Such a comparison is impossible in real-time for an in-person physical therapy, whereas the side-by-side visual panels in remote physical therapy provide a better feature in such cases. More generally, the Therapist and the Patients expressed that specific movement parameters such as range of motion, sway, reaction time and timing of movements were captured in real-time with our PTaaS interface. This immediate feedback to the Patient and Therapist was critical to modify the Patient movements via Therapist demonstration and verbal instructions. Lastly, both the Therapist and the patients showed a high level of enthusiasm and acceptance of the PTaaS interface despite the many underlying technological issues and changes made to the experiments as our study progressed.

\section{Controlled Network Experiments}

In this section, we describe our objective methods for collecting results that will provide an evaluation of the realtime data, computational resources, and network connectivity necessary for the proper functioning of the PTaaS interface. More specifically, we show how 'bad network' and 'good network' scenarios impact the network quality estimation, 


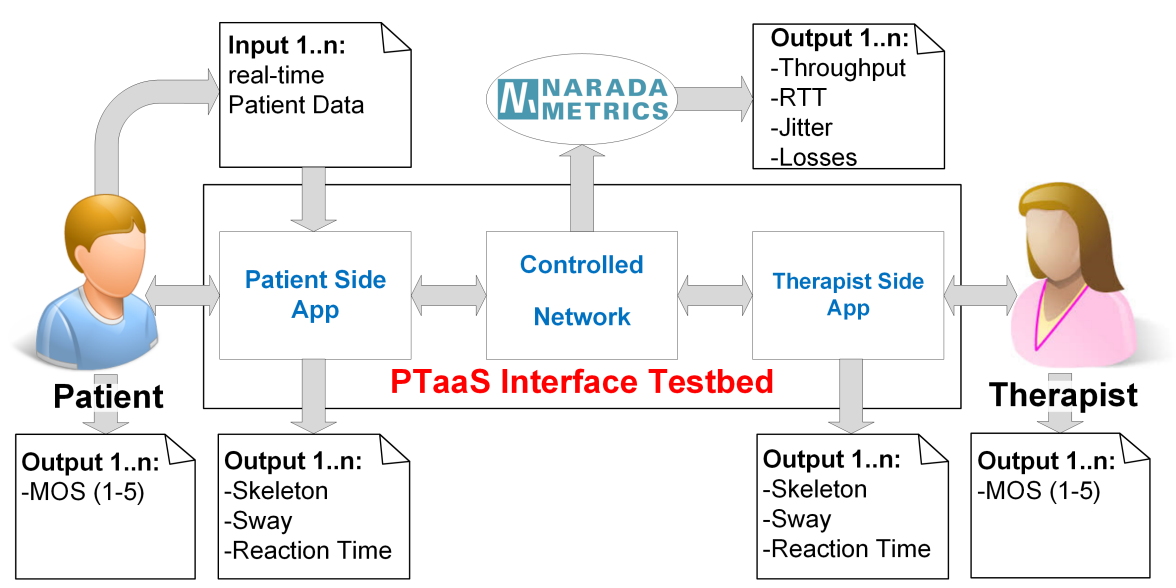

Fig. 8: General workflow of objective testing experiments

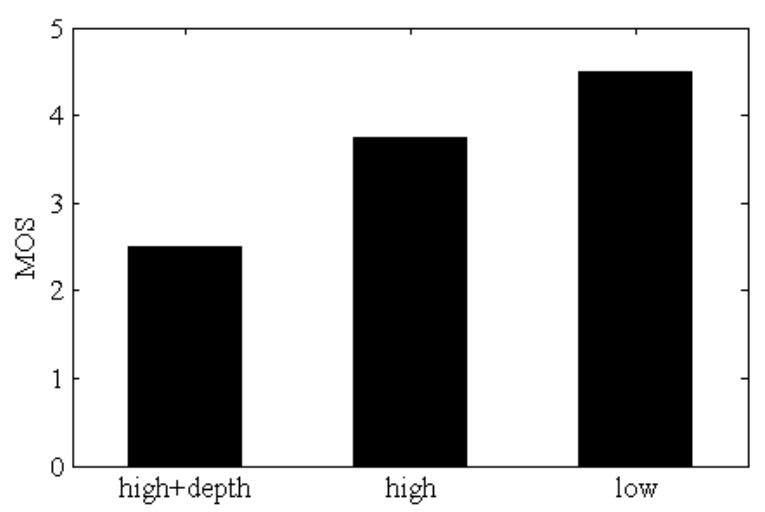

Fig. 9: Users' average MOS for different application modes, bad network scenario

as well as the PTaaS interface usability and effectiveness demands.

In our objective testing studies, our design of experiments involved three types of tests with different application modes of high or low resolution RGB video, and with or without depth data in the session as shown in Table IV. Each testing modes has two parts, one corresponds to an 'easy activity' (single leg stance) and the other corresponds to a 'difficult task' (tandem walk), and we conducted two tries per each activity. Moreover, for each experiment, we particularly capture the exercise activity-specific parameters, start time of activities using a timer, and the reaction time of the Patient in order to find the time delays associated with the data sent over the timer socket connection between the Patient and the Therapist. The reaction time in each test is particularly important to understand how much time it actually takes for a Patient to start a specific activity during a PTaaS exercise session after the timer starts.

The general workflow diagram of our objective testing experiments is shown in Figure 8. To synchronize both sides, we utilized the widely-used and conventional Network Time Protocol (NTP) [23]. However, as we will explain later, NTP did not provide enough precise time synchronization, especially for good network conditions; its worst-case accuracy is more than 100 milliseconds. Hence, we plan to explore other technologies such as Precise Time Protocol (PTP) [24] in our future PTaaS interface development iterations. In the interactions of the Therapist and the Patient using our PTaaS interface, we changed the application mode, and collected the various outputs from the data sources to perform a timesynchronized correlation analysis between the MOS rankings that indicate user satisfaction, and the application as well as network performance data. For a given network quality condition observed, we show how we update the weights of the network metrics by also considering the user opinion scores and categorize the condition as 'bad' (red bar in PTaaS interface) or 'good' (green bar in PTaaS interface).

1) Bad Network Scenario Results: In order to study the bad network scenario, we used the faulty network configuration described in Table I. We additionally used the Vyatta vRouter [25] at the software-level for the overlay network (Layer 2) path that further introduce overheads and performance bottlenecks for proper functioning of our PTaaS interface between the Patients in Kansas City and Therapist in Columbia. Our end-to-end available bandwidth in the overlay network channel as reported by Narada Metrics was a sustained $\approx 45$ Mbps.

Figure 9 shows the users' average MOS rankings (combined for Therapist and Patient) for the different application modes. We can see that the users found the low resolution mode to be more usable, and the high resolution with depth mode was practically unusable. The survey notes from the users indicated that the higher score for low resolution mode was due to the lower number of frame freeze events and inter-frame jitter, even though the picture quality was inferior in comparison to the other two high resolution application modes. It is expected that high resolution with depth mode that uses the most network resources has a larger impact on user experience, and thus suggests that it is not advisable to use high resolution with depth modes on medium to low network quality condition paths. Another interesting observation from the results in Figure 9 is that - in the cases where the PTaaS users may not always have high-bandwidth connections such as Google Fiber, there is an opportunity to consider other user-centered design and development approaches for the PTaaS interface to see how the video panels or sensor data visualizations can be improved to make the PTaaS session reasonably effective, even without a immersive experience expected on a high-bandwidth and low-latency network connection. 
TABLE IV: PTaaS Test Plan

\begin{tabular}{|l|l|l|l|}
\hline & Mode-1: Low Resolution & Mode-2: High Resolution & Mode-3: High Resolution with Depth \\
\hline Data Considered: & $\begin{array}{l}\text { RGB Video 640x480 + Kinect Audio + } \\
\text { Kinect Skeletal Data }\end{array}$ & $\begin{array}{l}\text { RGB Video 1280x960 + Kinect Audio } \\
\text { + Kinect Skeletal Data }\end{array}$ & $\begin{array}{l}\text { RGB Video 1280x960 + Kinect Audio } \\
\text { + Kinect Skeletal Data + Depth Data }\end{array}$ \\
\hline
\end{tabular}

To study the time synchronization issues in the bad network scenario, we start with high resolution with depth application mode. The main network metrics of throughput and RTT were in the range of average $30 \mathrm{Mbps}$ and $20 \mathrm{mlsec}$, respectively for the first and second activities. During the same time periods, the average jitter and loss measurements were $9 \mathrm{mlsec}$ (half the average RTT value; shows intermediate network congestion) and $75 \%$, respectively. For this mode, the ideal baseline throughput requirements are $100-120$ Mbps in average as obtained from our experiments in a controlled network setting with a $1 \mathrm{Gbps}$ connection. Figures 10 and 11 show throughput and RTT functions for two tries of the first activity. The three vertical dot lines with time labels on these figures are: (i) time when the therapist started the exercise activity (i.e., at 00.000 $\mathrm{sec}$ ), (ii) time when patient side interface started timer, and (iii) patient reaction time. We can notice that for both the tries, the network conditions had similar average statistics for most of the time, except they were a little bit higher for the throughput at the beginning of the second try. The resultant sway for the first activity (i.e., for single leg stance) had the value of 3 in average for both the tries as shown in Figure 12.

For this set of experiments, we update weights of high resolution with depth mode $w_{3, B}, w_{3, D}, w_{3, L}, w_{3, J}$, so that our function will be $\phi<0$ (poor); this is indicative of low user experience scores for $B_{a v}=30 \mathrm{Mbps}, D=20 \mathrm{mlsec}, L=75 \%$ and $J=9 \mathrm{mlsec}$. We also update the corresponding weights $w_{1, B}, w_{1, D}, w_{1, L}, w_{1, J}$ of the low resolution mode, so that our function will be $\phi \geq 0$ (good); this is indicative of the fact that the user experience is not relatively affected in this mode for $B_{a v}=20 \mathrm{Mbps}, D=20 \mathrm{mlsec}, L=68 \%$ and $J=16 \mathrm{mlsec}$. For this low resolution mode, the ideal baseline throughput requirements are $\approx 30 \mathrm{Mbps}$ in average as obtained from our experiments in a controlled network setting with a $1 \mathrm{Gbps}$ connection. Lastly, for the high resolution (without depth) mode, we update the corresponding weights $w_{2, B}, w_{2, D}, w_{2, L}, w_{2, J}$, so that our function will be $\phi=0$ (acceptable); this is indicative of the partly satisfactory user experience scores for $B_{a v}=20 \mathrm{Mbps}, D=20 \mathrm{mlsec}, L=70 \%$ and $J=11 \mathrm{mlsec}$.

2) Good Network Scenario Results: In order to study the good network scenario, we setup a closed-network with available bandwidth of $\approx 300$ Mbps via a VLAN extension (Layer 2) between two rooms within our MU Engineering Building between two different floors. Figure 13 shows the users' average MOS rankings marked separately for the Therapist and the Patients for the three application modes. We can see that the users found the high resolution (without depth) mode to be the most usable, and the lower resolution mode to be not as much satisfactory. In comparison to the MOS rankings of low resolution mode for the bad network scenario, we see the relative MOS effect, where the users had better experience with other modes and were willing to consider the low resolution mode to be not their desired mode for the PTaaS session. Interestingly, the high resolution with depth mode is quite similar to the high resolution mode, and the therapist ranking is slightly lower due to the more visual consumption

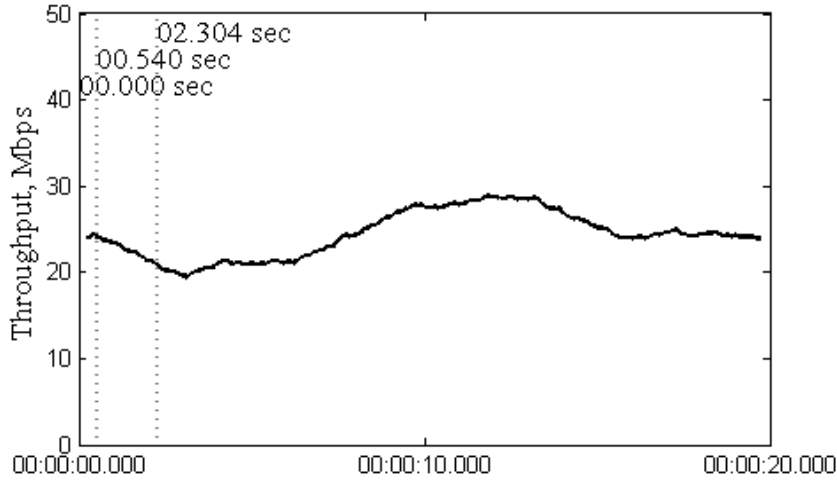

(a) First try

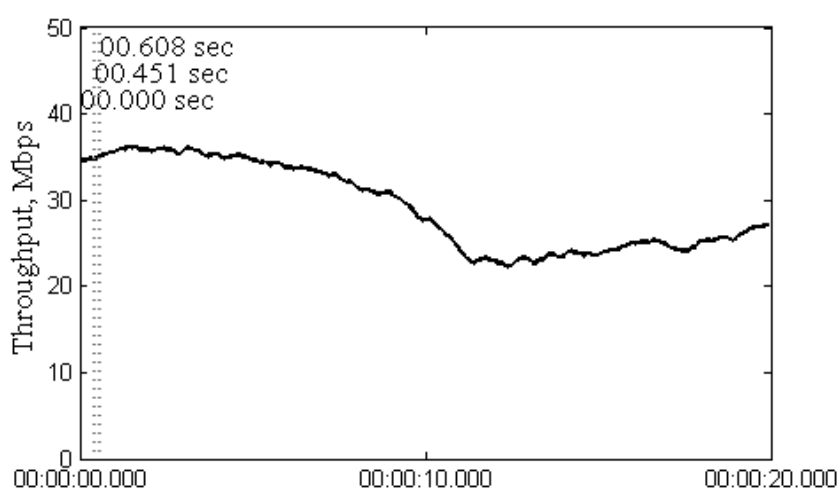

(b) Second try

Fig. 10: Throughput for first activity in high resolution with depth mode, bad network scenario

of the interface information necessary in the exercise activities. Thus, on a good network quality path, it is advisable to use both the high resolution, as well as the high resolution with depth modes as they provide a high ranking perceived user experience.

To study the time synchronization issues in the good network scenario, we start with the low resolution application mode. The main network metrics of throughput were $45 \mathrm{Mbps}$ in average for first activity, and $35 \mathrm{Mbps}$ for the second activity as seen in Figure 14. During the same time periods, the RTT values were $3 \mathrm{mlsec}$ and $2 \mathrm{mlsec}$ in average for first and second activities, respectively as seen in Figure 15. In addition, the jitter and loss measurements were $1 \mathrm{mlsec}$ and $0 \%$ for both activities. The resultant sway data of these activities are shown in Figure 16 (note that the patient and therapist data are shown on the same plot). We can observe that the shift between the two data in time is almost 0 for the first activity. Hence, we can say that the Therapist will see the Patient performance without any delay, similar to the case of in-person physical therapy user experience. However, the second result looks unintuitive, 


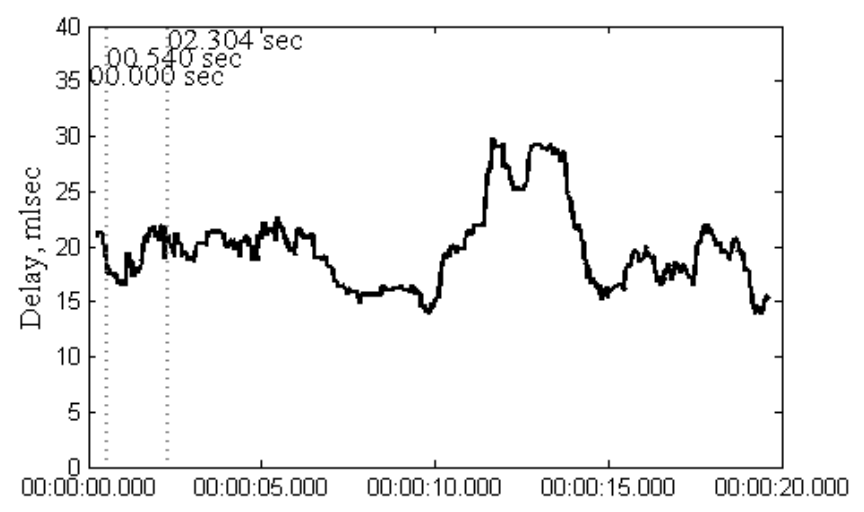

(a) First try

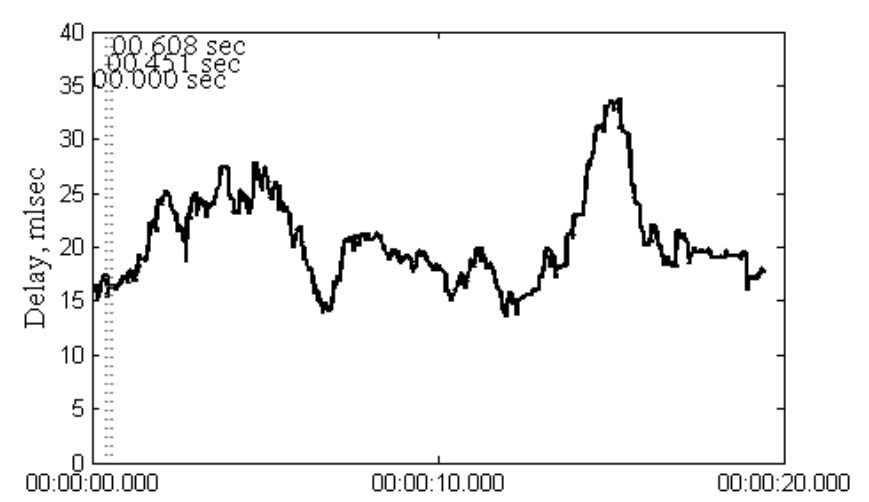

(b) Second try

Fig. 11: RTT for first activity in high resolution with depth mode, bad network scenario

as it seems that the Therapist observed the Patient movements before the Patient actually performed them. The cause of this phenomenon is due to the time synchronization accuracy issues within the NTP protocol, and thus we plan to further explore the opportunity to consider other time synchronization strategies for our PTaaS interface design and big data handling approaches in future work. Further, from Figure 17 for the high resolution with depth mode sway data, we can observe that similar trends occur due to the time synchronization accuracy issues within the NTP protocol.

For this set of experiments, we update weights of low resolution mode $w_{1, B}, w_{1, D}, w_{1, L}, w_{1, J}$, so that our function will be $\phi>0$ (good); this is indicative of the relatively lower user experience scores with equal sway trends on both sides, which are still in the good performance range for $B_{a v}=35 \mathrm{Mbps}, D=3 \mathrm{mlsec}, L=0 \%$ and $J=1 \mathrm{mlsec}$. We also update the corresponding weights $w_{3, B}, w_{3, D}, w_{3, L}, w_{3, J}$ for the high resolution with depth mode, so that our function will be $\phi>0$ (good); this is indicative of the high user experience scores with equal sway trends on both sides, which are in the good performance range for $B_{a v}=100 \mathrm{Mbps}, D=$ $6 \mathrm{mlsec}, L=0 \%$ and $J=2 \mathrm{mlsec}$. Lastly, for the high resolution (without depth) mode, we update the corresponding weights $w_{2, B}, w_{2, D}, w_{2, L}, w_{2, J}$, so that our function will be $\phi>0$ (good); this is indicative of the highest user experience scores recorded int his mode with equal sway trends on

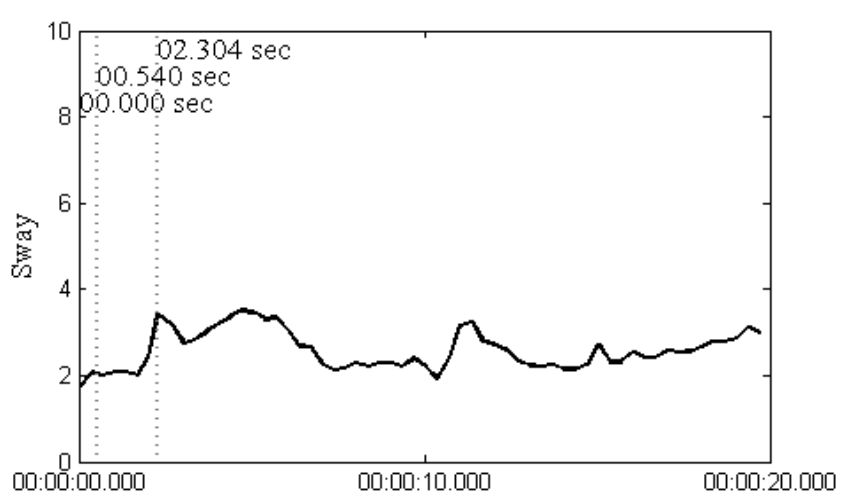

(a) First try

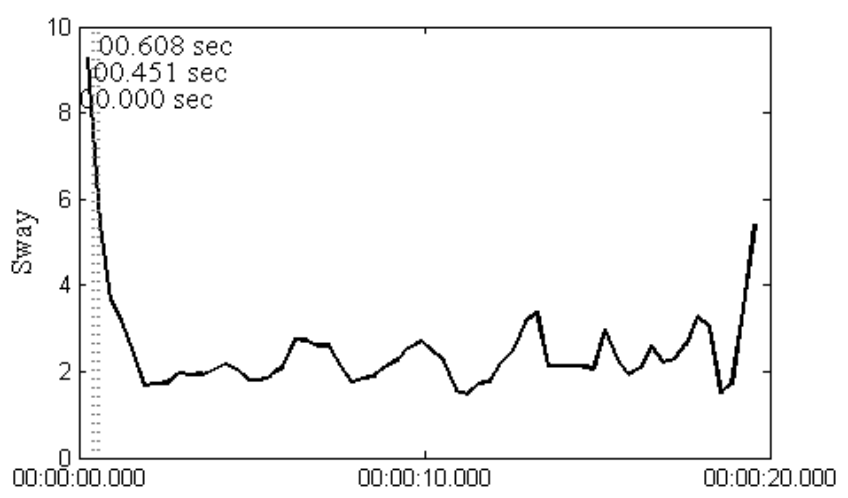

(b) Second try

Fig. 12: Patient sway for first activity in high resolution with depth mode, bad network scenario

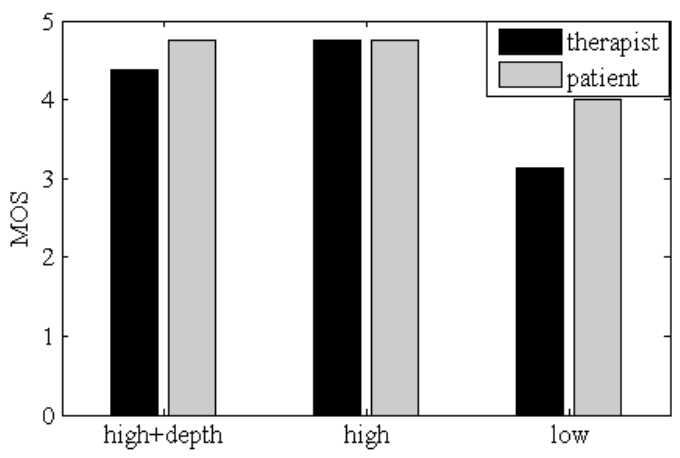

Fig. 13: Users' average MOS for different application modes, good network scenario

both sides, which are in the good performance range for $B_{a v}=50 \mathrm{Mbps}, D=3 \mathrm{mlsec}, L=0 \%$ and $J=1 \mathrm{mlsec}$.

\section{PTaAs Big Data Ecosystem}

Our PTaaS interface is one of the major applications within a practical health care environment that is coupled with Big Data analytics. Our study findings through actual network and controlled network scenarios provided valuable insights on the network, compute and storage resource calibration issues, as well as the performance troubleshooting issues that involve 


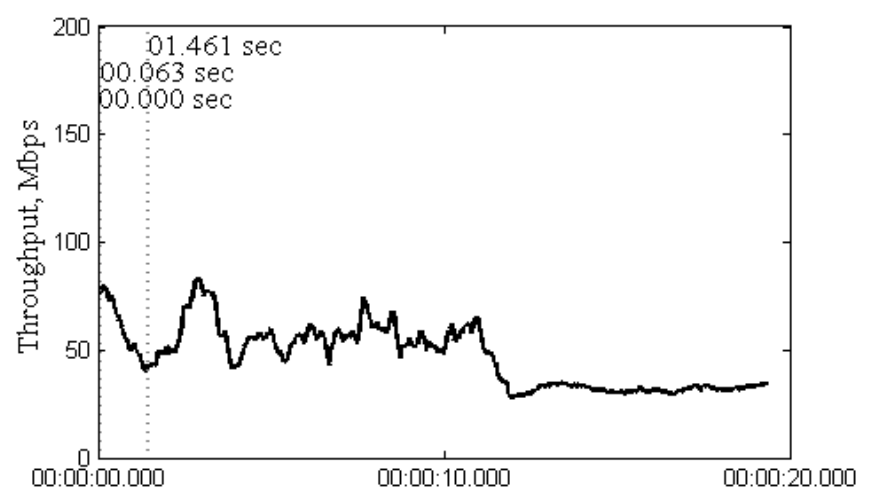

(a) First activity

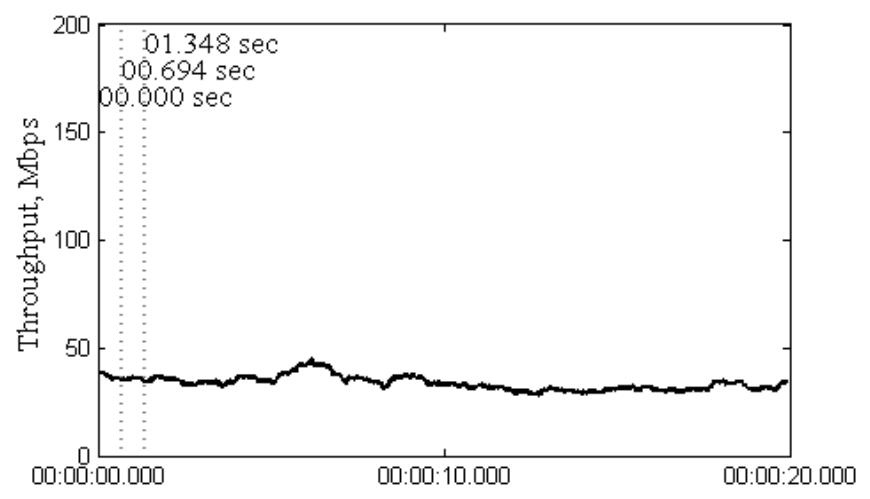

(b) Second activity

Fig. 14: Throughput for low resolution mode, good network scenario

network effects as well as time synchronization accuracy. Our future work is to explore the architectures for PTaaS that could be applied to other similar personalized healthcare services that are remotely delivered at-scale (for hundreds or even thousands of customers simultaneously) through a cloud computing platform and various Big Data analytics tools in a reliable, secure and cost-effective manner.

Figure 18 illustrates the various architecture components necessary for at-scale delivery of PTaaS within an actual clinical environment. We envisage two layers of services that support the PTaaS through RESTful APIs that are commonly used in cloud platforms viz.,: (i) Secure Compute and Network Services, and (ii) Data Services. In the first layer, components are needed for different processing pipelines that handle the workflow as well as security issues for data-in-motion (e.g., SSL), and the access control as well as encryption to the data-at-rest. The access control is possible through Federated Identity and Access Management frameworks that have web interfaces for the owners of the PTaaS system to be able to add, delete or modify permissions of the different data query and analysis tasks. A major challenge in the infrastructure configuration for secure services can be related to the compliance issues (i.e., pertaining to FISMA Moderate/HIPPA) that are paramount in the health care related technology infrastructures. In the second layer, the components are essentially related to data services that operate in virtualized storage environments

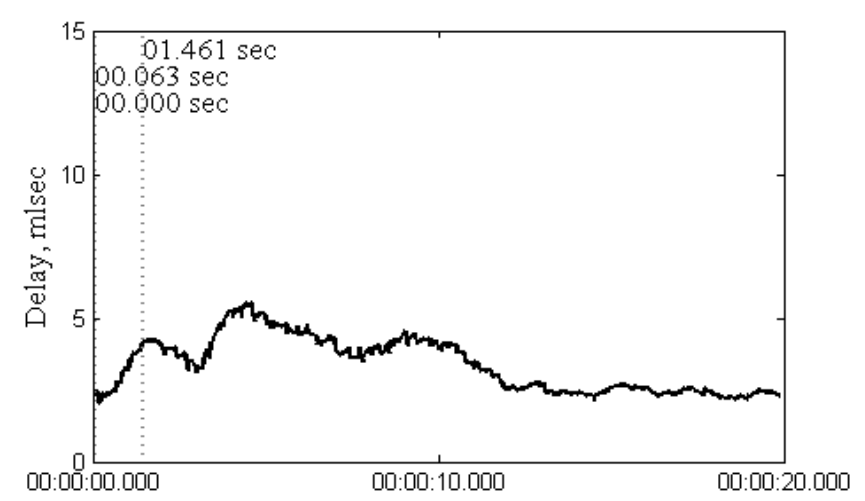

(a) First activity

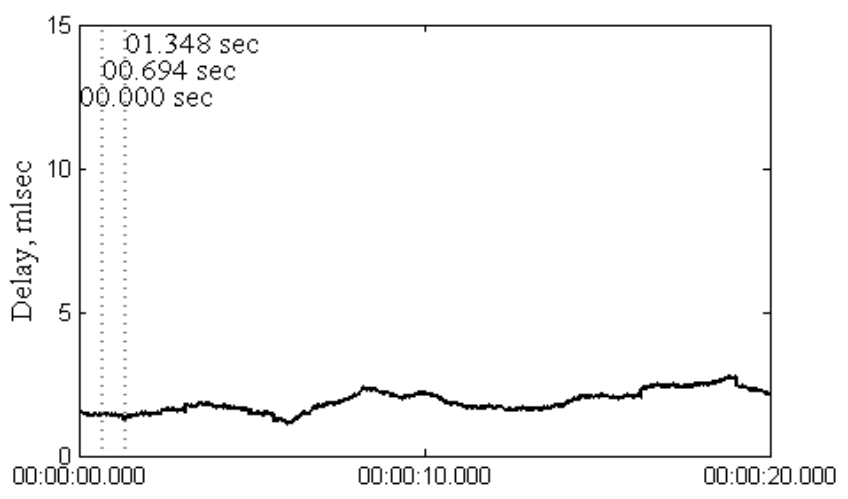

(b) Second activity

Fig. 15: RTT for low resolution mode, good network scenario

that are again federated across multiple private/public cloud platforms. Last, but definitely not the least, the deep and continuous monitoring with frameworks such as Narada Metrics as shown in this study, are fundamental to query data from multiple sources, and feed it to tools that perform the Big Data analytics in a synchronous or asynchronous manner as per the health care use case needs.

\section{CONCLUSION AND FUture WORK}

In this paper, we presented a 3D real-time interactive system viz., PhysicalTherapy-as-a-Service (PTaaS) that can be used by a Therapist to remotely monitor Patient performance within an exercise balance assessment program over high-speed network connections. Using the features in the PTaaS interface, we showed how the Therapist was able to monitor Patient status, offer verbal, auditory and visual cues to perform correct exercise movements, all through a synchronous Big Data analytics approach we adopted. Our veracity analytics approach correlated the high volume, velocity, and variety of data by addressing challenges relating to network configuration and time synchronization within wide-area overlay network paths between the Therapist and the Patient. Our results showed that we were able to distinguish scenarios where there was bias due to network quality effects, and thus enabled the Therapist to successfully quantify and assess balance of the senior study participants we recruited in private homes. 


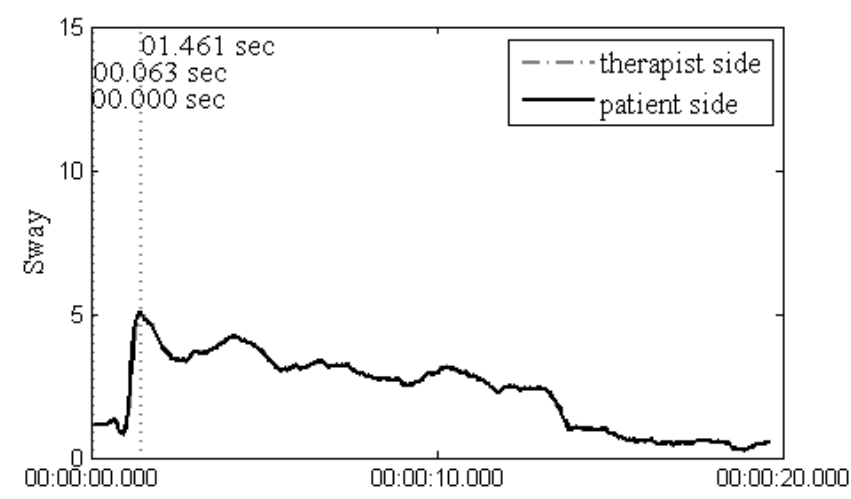

(a) First activity

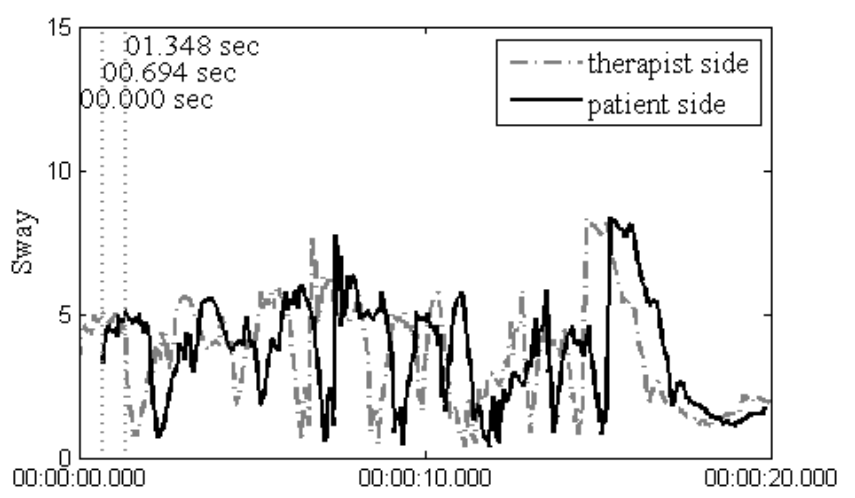

(b) Second activity

Fig. 16: Sway for low resolution mode, good network scenario

We conducted experiments and described results in both qualitative and objective testing cases, which showed how the PTaaS interface is usable and effective for remote therapy involve real-time analysis of specific movement parameters such as range of motion, sway, reaction time and timing of movements. The real-time analysis fostered immediate feedback to the Patient and Therapist, who then used the feedback to modify the exercise movements via further Therapist demonstration and verbal instructions. Both the user satisfaction and interaction effectiveness information captured through opinion scores showed a high-level of enthusiasm and acceptance by the Patients and Therapist, despite technological issues and changes made to the system as the study progressed.

Our future work is to further prove the clinical validity of our PTaaS application by extensively testing and refining our system with a larger groups of Patients and Therapists. Our planned future work is to also evaluate our system with gold standard physical therapy assessment systems that use force-plates and accelerometers. Further, our PTaaS interface integration plans with a Big Data eco-system can decrease the frequency with which Patients would have to travel to a clinic, while still being able to receive one-on-one interactive care from a Therapist. Ultimately, our efforts for remote monitoring with our PTaaS interface are well suited for transition of a Patient to total independence with their home exercise program, as part of a therapeutic program or even within health and wellness programs relating to the area of fall prevention.

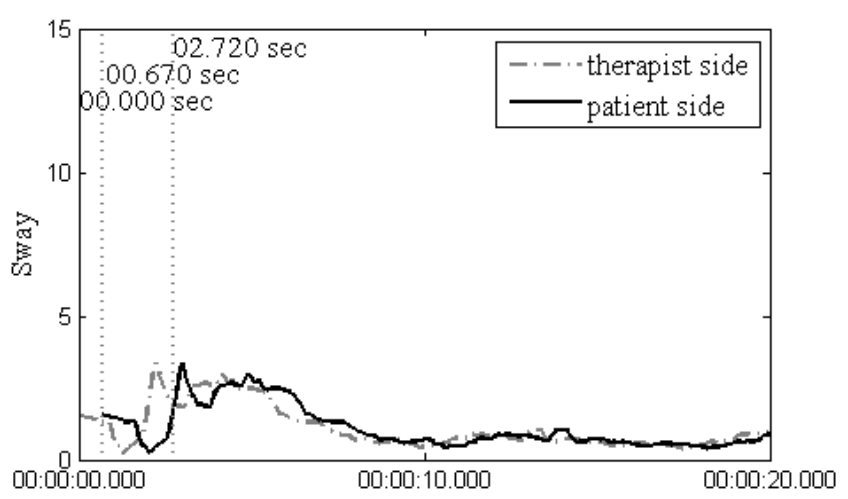

(a) First activity

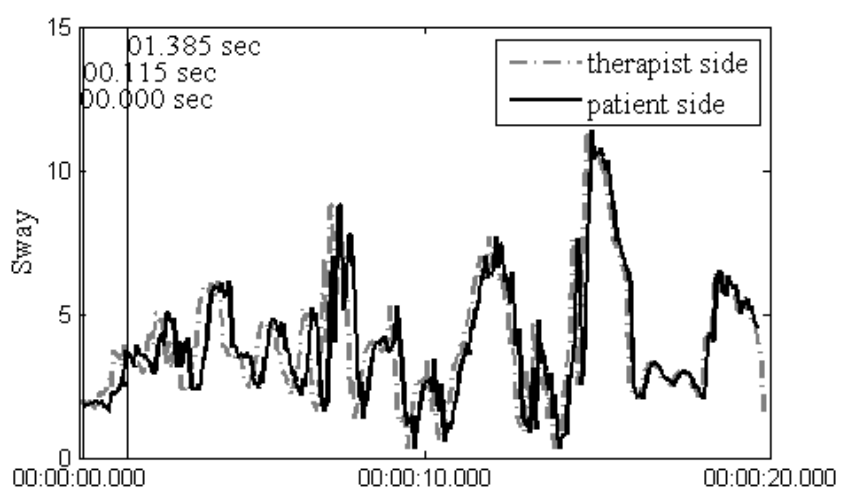

(b) Second activity

Fig. 17: Sway for high resolution with depth mode, good network scenario
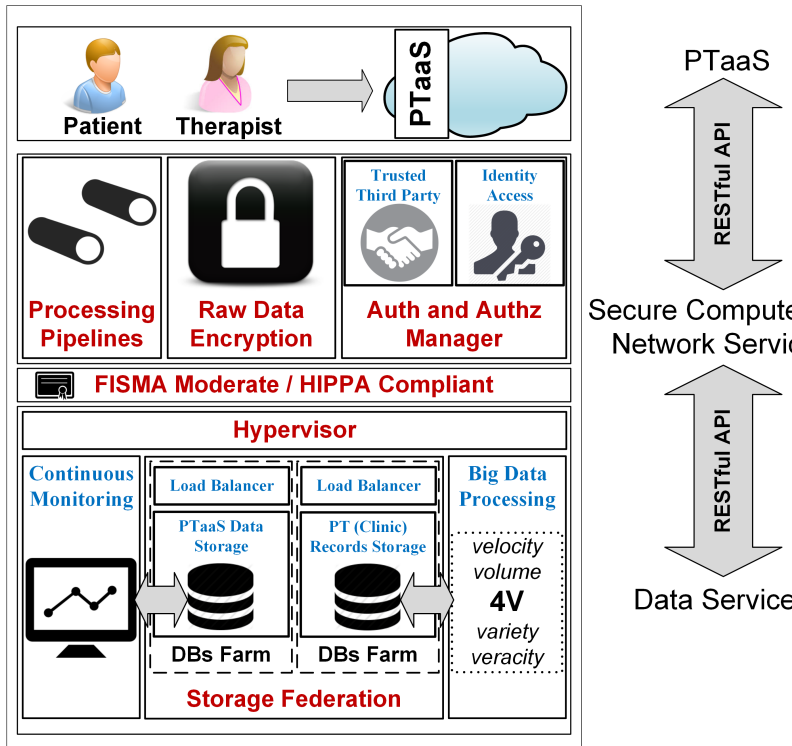

Secure Compute and Network Services

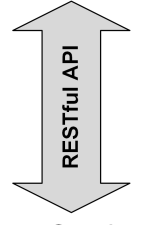

Data Services

Fig. 18: Big Data architecture for PTaaS at-scale 


\section{REFERENCES}

[1] P. Calyam, L. Kumarasamy, C. Lee, F. Ozguner, "Ontology-based Semantic Priority Scheduling for Multi-domain Active Measurements", Springer Journal of Network and Systems Management (JNSM), 2014. (http://www.naradametrics.com)

[2] M. Berman, J. Chase, et. al., "GENI: A Federated Testbed for Innovative Network Experiments", Elsevier Computer Networks, 2014.

[3] US Ignite Initiative - http://www.us-ignite.org

[4] C. Camporesi, M. Kallmann, J. Han, "VR Solutions for Improving Physical Therapy", Proc. of IEEE Virtual Reality Conference, 2013.

[5] D. Tacconi, R. Tomasi, C. Costa, O. Mayora, "A System for Remote Orthopedics Rehabilitation", Proc. of IEEE PervasiveHealth, 2013.

[6] J. Fasola, M. Mataric, "A Socially Assistive Robot Exercise Coach for the Elderly", Journal of Human-Robot Interaction, Vol. 2, No. 2, pp. 3-32, 2013.

[7] S. Obdr?lek, G. Kurillo, E. Seto, R. Bajcsy, "Architecture of an Automated Coaching System for Elderly Population", Proc. of MMVR, 2013.

[8] Y. Chen, Y. Hung, "Using Real-time Acceleration Data for Exercise Movement Training with a Decision Tree Approach", Proc., IEEE Conference on Machine Learning and Cybernetics, 2009.

[9] K. Huang, P. Sparto, S. Kiesler, D. Siewiorek, A. Smailagic, "iPod for Home Balance Rehabilitation Exercise Monitoring", Proc., IEEE Symposium on Wearable Computers, 2012.

[10] L. Mize, R. Klenke, J. McCollum, "Snapshot Capture from Live Highdefinition Video Stream for Transmission over Low-bandwidth Data Link", Proc. of IEEE SoutheastCon, 2010.

[11] L. Tan, S. Lau, T. Chong, "Enhanced Compression Scheme for Highlatency Networks to Improve Quality of Service of Real-time Applications", Proc. of Asia-Pacific Symposium on Information and Telecommunication Technologies, 2010.

[12] M. Shah, K. Jinman, M. Khadra, D. Feng, "Enhancing Home Area Networks to Facilitate Telehealth Services: Test-bed Scenario with Videoconsultation-calls (VCC)", Proc. of IEEE International Conference on Orange Technologies, 2014.

[13] P. Calyam, A. Kalash, R. Gopalan, S. Gopalan, A. Krishnamurthy, "RICE: A Reliable and Efficient Remote Instrumentation Collaboration Environment", Journal of Advances in Multimedia, 2008.

[14] J. Heer, S. Kandel, "Interactive Analysis of Big Data", ACM XRDS: Crossroads Magazine, 2012.

[15] G. Jung, N. Gnanasambandam, T. Mukherjee, "Synchronous Parallel Processing of Big-data Analytics Services to Optimize Performance in Federated Clouds", Proc. of IEEE Cloud, 2012.

[16] A. Jacobs, "The Pathologies of Big Data", Communications of the ACM, Vol. 52, No. 8, pp. 36-44, 2009.

[17] P. Gui, Y. Qin, C. Hongmin, Z. Tinghui, Y. Chun, "Accurately calibrate kinect sensor using indoor control field", IEEE Workshop on Earth Observation and Remote Sensing Applications, 2014.

[18] J. Chow, D. Lichti, "Photogrammetric Bundle Adjustment With SelfCalibration of the PrimeSense 3D Camera Technology: Microsoft Kinect", Proc. of IEEE Access, 2013.

[19] A. Mishra, M. Skubic, C. Abbott, "Development and Preliminary Validation of an Interactive Remote Physical Therapy System", Proc. of IEEE Engineering in Medicine and Biology Society, 2015.

[20] E.Stone, M. Skubic, "Fall Detection in Homes of Older Adults Using the Microsoft Kinect", IEEE Journal of Biomedical and Health Informatics, Vol. 19, No. 1, pp. 290-301, 2015.

[21] E. Stone, M. Skubic, "Unobtrusive, Continuous, In-Home Gait Measurement Using the Microsoft Kinect", IEEE Transactions on Biomedical Engineering, Vol. 60, No. 10, pp. 2925-2932, 2013.

[22] M. Skubic, R. Guevara, M. Rantz, "Automated Health Alerts Using InHome Sensor Data for Embedded Health Assessment", IEEE Journal of Translational Engineering in Health and Medicine, Vol. 3, pp. 1-11, 2015.

[23] D. Mills, "Network Time Protocol (NTP), IEEE Trans. on Communications, Vol. 39, No. 10, pp. 1482-1493, 1991.

[24] A. Vallat, D. Schneuwly, "Clock Synchronization in Telecommunications via PTP (IEEE 1588)", Proc. of IEEE Frequency Control Symposium, 2007.

[25] B. Gillian, "VYATTA: Linux IP Routers", 2007. (http://freedomhec.pbworks.com/f/linux_ip_routers.pdf) 